\title{
Implant-Tolerant Orthopaedic Measurements for Post-Operative Radiographs of the Lower Limbs
}

\author{
André Gooßen ${ }^{1,2}$, Georg M. Weber ${ }^{1,2}$, Thomas Pralow $^{2}$, Rolf-Rainer Grigat ${ }^{1}$ \\ ${ }^{1}$ Vision Systems, Hamburg University of Technology \\ ${ }^{2}$ Philips Healthcare, Diagnostic X-Ray, Hamburg \\ andre.goossen@tu-harburg.de
}

\begin{abstract}
In this work we present a method for automated orthopaedic measurements for patients that have undergone a partial or full joint replacement in the lower limbs. In contrast to previously published approaches for partially occluded objects, we deal with objects were the major part of the contour is missing, namely the epiphyses of the long bones in the lower limbs, that have been replaced in large parts by artificial joint implants of varying appearance. We present an approach based on the automatic detection and segmentation of implants and a robust adaptation of a segmentation technique based on deformable models. We evaluated our method on a set of clinical images and achieve an accuracy of $0.6^{\circ}$ for angles and $1.3 \mathrm{~mm}$ for lengths measurements while significantly reducing assessment time and eliminating user interaction.
\end{abstract}

\section{Introduction}

Joint replacement surgery has become a standard procedure in orthopaedics. In Germany, according to the German Federal Association of Medical Technology, more than 400,000 artificial hip and knee joints are implanted each year [1]. To rate the success of a replacement surgery it is necessary to measure several quantities on a pre- as well as a post-operative radiograph of the lower limbs.

There exist various methods for segmentation of the bone structure in digital radiographs. In a recent publication, Gooßen et al. [2] achieve an average accuracy of $0.5 \mathrm{~mm}$ when segmenting the joints of the lower limbs. However, as with any of the previously published method, their approach does not incorporate post-operative segmentation after joint replacement.

Dong et al. [3] evaluated their hybrid approach, based on geometric models and shape priors, occluding small fractions of the bone contour. But an implant typically replaces major parts of the bone and does therefore not match their presumption. Though there exists a model-based approach for the segmentation of total hip joints replacements (THR) by Kotcheff et al. [4], it only works up to a certain degree of similarity between the trained and actual prostheses. The German Federal Association of Medical Technology, however, reports more than 200 different types of prostheses for the hip joint alone [1], each available in different sizes, ruling out any model-based technique. 


\section{Materials and Methods}

In order to tolerate implants when measuring orthopaedic quantities within the lower limbs, we developed an approach consisting of two complementing stages. The first one robustly detects the presence of implants and segments them with pixel accuracy. Subsequently, a second step adapts the deformable templates used for segmentation in order to avoid the implant structure and precisely delineate the remaining bone contours.

\subsection{Automatic Implant Segmentation}

Implants in radiographs showcase a distinct sharpness of edges and homogeneous brightness due to the high absorption of its materials. We exploit these features by creating binary images $B_{\leftrightarrow}, B_{\uparrow}$, containing pixels enclosed by strong horizontal and vertical edges, respectively. Another binary image, $B_{\text {hist }}$, is created via histogram-adaptive thresholding of the input image. Intersecting these binary images and morphologically eroding with structure element $v$ for leakage avoidance yields the candidate image $B_{\text {seed }}=\left(B_{\leftrightarrow} \cap B_{\uparrow} \cap B_{\text {hist }}\right) \ominus v$.

To identify connected areas in $B_{\text {seed }}$ we combine Region Growing with the Level-Set cost function of Malladi et al. [5]

$$
g=\frac{1}{1+\left|\nabla\left(G_{\sigma} * I\right)\right|}
$$

as the stopping criteria to benefit from the former speed and the latter accuracy.

To save processing time, preliminary processing up to this point utilizes a lower level of a Gaussian pyramid, hence the implant segmentation result lacks precision. To maximize accuracy, the now known location of the implant borders are utilized in a further local adaptive thresholding procedure. Herein Otsu's algorithm [6] is applied to a small window which is shifted along the implant outline in $I$ to produce refined implant edges in image $B_{\text {Otsu }}$. Since local image content can display several general brightness classes (implant, dense bone tissue, soft tissue, and direct radiation) we found that it is advantageous to perform the thresholding with three classes. Fig. 1 displays the intermediate images resulting in the implant delineation $\gamma$ overlayed onto the original image $I$.

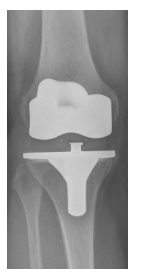

(a) $I$

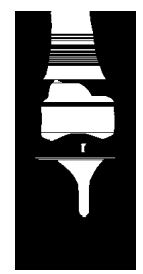

(b) $B_{\leftrightarrow}$

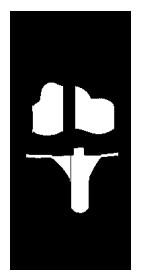

(c) $B_{\uparrow}$

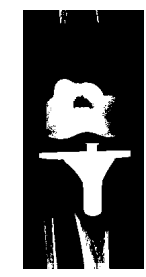

(d) $B_{\text {hist }}$

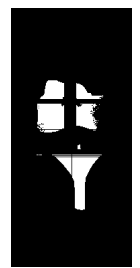

(e) $B_{\text {seed }}$

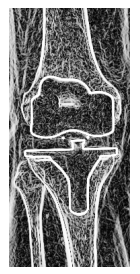

(f) $g$

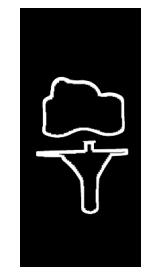

(g) $B_{\text {Otsu }}$

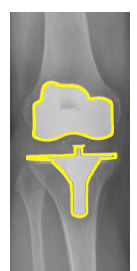

(h) $\gamma$

Fig. 1. Implant segmentation from (a) original image to (h) overlayed implant. 


\subsection{Bone Structure Segmentation in Presence of Implants}

For the joint segmentation in pre-operative images we trained dedicated deformable template models on over 100 radiographs of patients without joint replacement. With $\overline{\boldsymbol{x}}$ denoting an average representation of a point distribution model and $\boldsymbol{P}$ denoting the corresponding modes of variation we can approximate any shape $\boldsymbol{x}$ by adding a linear combination of eigenvectors to the mean shape, i.e.

$$
\boldsymbol{x} \approx \overline{\boldsymbol{x}}+\boldsymbol{P b}
$$

with $\boldsymbol{b}$ denoting the shape coefficients. When approximating an unknown shape $\hat{\boldsymbol{x}}$, we determine the model parameters $\boldsymbol{b}$ that minimize the error

$$
\Delta=(\hat{\boldsymbol{x}}-(\overline{\boldsymbol{x}}+\boldsymbol{P b}))^{T} \boldsymbol{W}(\hat{\boldsymbol{x}}-(\overline{\boldsymbol{x}}+\boldsymbol{P b})), \quad \boldsymbol{W}=\operatorname{diag}\left(w_{1}, \ldots, w_{2 n}\right)
$$

between a shape candidate $\hat{\boldsymbol{x}}$ and the shape $\boldsymbol{x}$, generated by using Eq. (2). The weights $w_{1}, \ldots, w_{2 n}$ control the influence of a specific landmark $\left(x_{i}, y_{i}\right)$ [2].

In order to connect the trained shape to the image data we also have to learn the local appearance around each of these landmarks. For this purpose we extract a sampling vector $\boldsymbol{s}_{i, j}$ perpendicular to the local shape tangent for each landmark. Similar to the training of the shape we derive the mean appearance $\bar{s}_{i}$ for all the models of the training set and the empirical covariance matrix, estimated by $\boldsymbol{S}_{i}$.

We locate an initial position of the template model using a Generalized Hough Transform (GHT) [7] and iterate on multiple scales until convergence. This algorithm, however, fails for artificial objects within the capture range of the template model and thus has to be adapted to reliably segment joints with implants.

To avoid the implant edges from attracting the shape model we check whether the search vector $\hat{\boldsymbol{s}}_{i}$ overlaps the segmented implant region $\gamma$. For any candidate $\left(\hat{x}_{i}, \hat{y}_{i}\right)$ with such a distorted search vector we set the weights

$$
w_{i}=w_{n+i}= \begin{cases}0, & \hat{\boldsymbol{s}}_{i} \cap \gamma \\ 1, & \text { else }\end{cases}
$$

As we use a coarse-to-fine approach with increasing image resolution, candidates that have been disabled on a coarser level might get a valid weight $w_{i}$ on

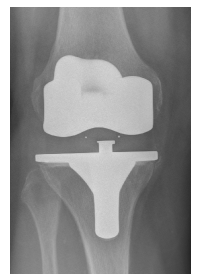

(a) Original

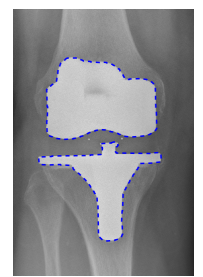

(b) Implant

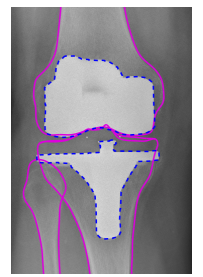

(c) ASM result

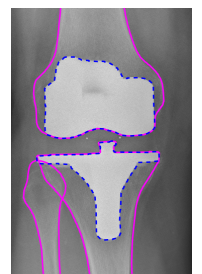

(d) Fused contour

Fig. 2. Fusion of ASM and implant segmentation. 
a finer resolution and contribute to the delineation. Thus we achieve maximum accuracy while maintaining the robustness against artificial objects.

After the deformable template model has converged, the bone shape is merged with the implant contour. In order to do this, intersection points between the fitted shape and the implant contour need to be identified. In the tibial case the closest landmarks of the fitted shape are dragged to the coordinates of maximum lateral and medial elongation of the lower knee implant. For the femur, the lateral and medial intersections between fitted shape and implant contour are localized. In a last processing step, the incorrectly segmented portions of the fitted shape are replaced by the respective implant borders (see Fig. 2).

We evaluated our method on 20 long-leg radiographs of the same patients prior to and after joint replacement surgery using standard orthopaedic measurements for the mechanical knee axis assessment (nomenclature according to Paley \& Herzenberg [8]). These measurements serve as guidance for therapy planning prior to and success rating after surgical treatment.

\section{Results}

For the implant segmentation we achieve a true positive rate (TPR) of $97.5 \%$. Together with an average curve-to-curve error of $0.5 \mathrm{~mm}$ for the bone segmentation these accurate delineations result in precise measurements with a mean deviation of $0.6^{\circ}$ and $1.3 \mathrm{~mm}$ for angle and length measurements, respectively. Fig. 3 depicts automatically derived measurements on a pre-operative radiograph and the post-operative examination of the same patient. We evaluated the proposed method on 20 radiographs containing lower limbs. Half of the set consists of pre-operative and the other half of the same patients post-operative imagery. Fig. 4 compares our results to inter-observer variability according to a dedicated study by Gordon et al. [9] for manual measurement and to our own study using a computer assisted approach.
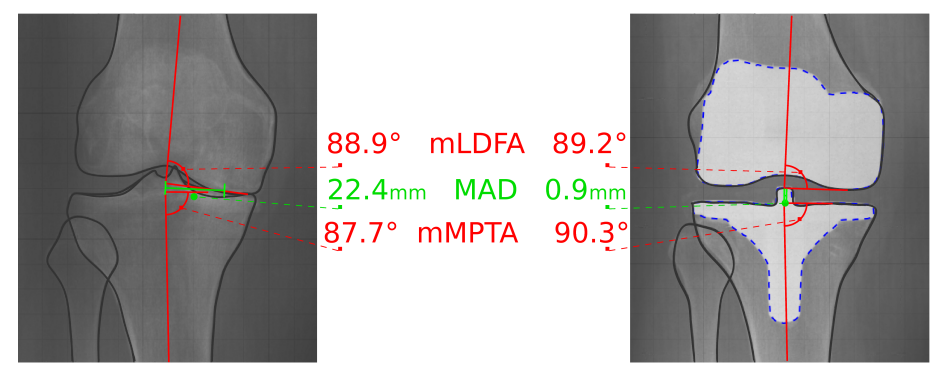

Fig. 3. Automatically derived pre- and post-operative knee joint morphometry of the same patient (nomenclature according to Paley \& Herzenberg [8]). 
Fig. 4. Accuracy of pre- and post-operative measurements. The bars depict mean deviations as well as $95 \%$ confidence intervals. Shaded bars correspond to inter-observer accuracy. The pre-operative angular measurements show poor performance because patients developed heavy arthritis leaving no visible border between femur and tibia.

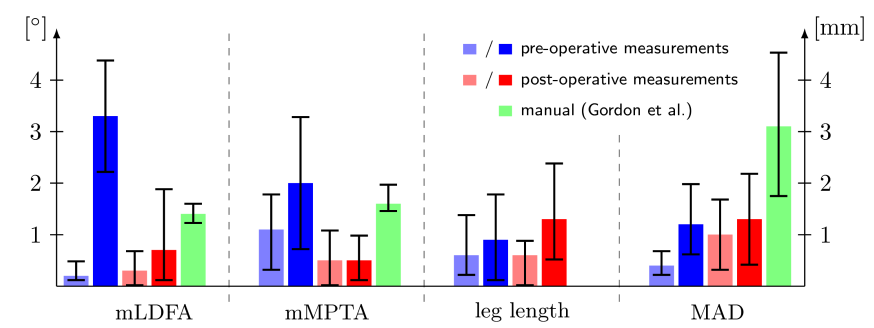

\section{Discussion}

Our results indicate that the proposed automatic method on average outperforms manual measurement. In all cases, except for the pre-operative mMPTA, the mean error of automatic assessment is superior to manual derivation. Compared to computer-assisted measurements, automatic results achieve comparable accuracy, except for the pre-operative measurements of mechanical angles. These are tampered by strong arthritis in the knee joints with no joint space and thus overlapping borders of femur and tibia. Our method reduces the processing time by a factor of 20-35 to $20 \mathrm{~s}$ compared to $394 \mathrm{~s}$ and $706 \mathrm{~s}$ for computer-assisted and manual measurements [10], respectively, and does not require user interaction.

\section{References}

1. Beeres M. Medienservice-Papier zum künstlichen Gelenkersatz. Bundesverband Medizintechnologie, 2009; 2009.

2. Gooßen A, Hermann E, Gernoth T, et al. Model-based lower limb segmentation using weighted multiple candidates. Proc BVM. 2010; p. 376-80.

3. Dong X, Zheng G. Automatic extraction of femur contours from calibrated x-ray images: A Bayesian inference approach. In: Proc Biomed Imag; 2008. p. 57-60.

4. Kotcheff ACW, Redhead A, Taylor CJ, et al. Shape model analysis of THR radiographs. Proc ICPR. 1996;4:391-5.

5. Malladi R, Sethian JA, Vemuri BC. Shape modeling with front propagation: a level set approach. IEEE Trans Pattern Anal Mach Intell. 1995;17:158-75.

6. Otsu N. A threshold selection method from gray-level histograms. IEEE Trans Syst Man Cybern. 1979;9(1):62-6.

7. Ruppertshofen H, Lorenz C, Beyerlein P, et al. Fully automatic model creation for object localization. Proc BVM. 2010; p. 331-5.

8. Paley D, Herzenberg JE. Principles of Deformity Correction. Springer; 2002.

9. Gordon JE, Chen RC, Dobbs MB, et al. Interobserver and intraobserver reliability in the evaluation of mechanical axis deviation. J Pediatr Orthop. 2009;29(3):281-4.

10. Hankemeier S, Gosling T, Richter M, et al. Computer-assisted analysis of lower limb geometry: higher intraobserver reliability compared to conventional method. Comput Aided Surg. 2006;11:81-6. 